\title{
Pluto's Extended Atmosphere: An Escape Model and Initial Observations
}

\author{
JoHN T. Clarke \\ Department of Atmospheric, Oceanic, and Space Sciences, The University of Michigan, Ann Arbor, Michigan 48109-2143, \\ S. Alan Stern' \\ CASA, Campus Box 391, University of Colorado, Boulder, Colorado 80309
}

AND

Laurence M. Trafton

Astronomy Department, University of Texas at Austin, Austin, Texas 78712

Received April 1; revised October 4, 1991

We have calculated the rates of production and hydrodynamic outflow of atomic hydrogen resulting from the photodissociation of methane in the upper atmosphere of Pluto. Under the present near-perihelion conditions this yields an extended cloud of $\mathbf{H}$ around Pluto which is likely to be the most easily observable signature of Pluto's extended atmosphere, and thereby provide information on the extent, escape rate, and composition of Pluto's upper atmosphere. We have also performed initial observations with the IUE attempting to detect the H Ly $\alpha$ emission from the extended H cloud, which we use to derive upper limits to the cloud properties as a function of the cloud extent. 1992 Academic Press, Inc.

\section{INTRODUCTION}

There have been major advances in our understanding of Pluto's atmosphere over the last few years, driven in part by observations of the mutual eclipses of Pluto and Charon and a stellar occultation, and in part by theoretical calculations inspired by ground-based observations (cf. the review by Trafton 1989 and references therein). Pluto's atmosphere is believed to be generated by sublimation vapor pressure from the surface, and only $\mathrm{CH}_{4}$ has been spectroscopically identified (e.g., cf. Stern and Trafton 1984). Although this methane is not spectrally discriminated into solid and gas phase components, the occultation-derived reference level pressure is $14 \pm 4$ mbar (Elliott et al. 1989, Hubbard et al. 1988). The bulk atmosphere is thought to be a mixture of $\mathrm{CH}_{4}$ and a heavier

\footnotetext{
${ }^{1}$ Present address: Div. 15/Space Sciences, Southwest Research Institute, 6220 Culebra Road, San Antonio, TX 78238.
}

gas (either $\mathrm{CO}$ or $\mathrm{N}_{2}$ ) required both to explain Pluto's occultation lightcurve (Hubbard et al. 1990) and more recently by the analogy to the $\mathrm{CH}_{4} / \mathrm{N}_{2}$ atmosphere of Triton. The stellar occultation profile is consistent with either a pure $\mathrm{CH}_{4}$ atmosphere at $67 \mathrm{~K}$ and a low-lying haze layer (Elliot et al. 1989) or a solar-heated atmosphere with a temperature gradient peaking near $105 \mathrm{~K}$ (Hubbard et al. 1990). Whatever the exact composition and temperature of the atmosphere, it must be escaping rapidly into space at the temperatures experienced at the present nearperihelion distance of Pluto (Hunten and Watson 1982). Total escape rates on the order of $10^{28} \mathrm{sec}^{-1}$ have been estimated depending on the atmospheric model (Trafton et al. 1988a, McNutt 1989), requiring a volatile reservoir source to replenish the atmosphere over geologic time.

Pluto's atmosphere is one of the few examples of active hydrodynamic escape in the Solar System today, and hydrodynamic escape is thought to have strongly influenced the early evolution of the atmospheres of the terrestrial planets. Two of the outstanding questions about Pluto's atmosphere are its bulk composition (and hence the mixing ratio of $\mathrm{CH}_{4}$ ) and the extent and escape rate of the upper atmosphere. Based on existing remote observations, these parameters are difficult to pin down. In this paper we present calculations and initial observations of what we believe is the most easily observable diagnostic emission from Pluto's upper atmosphere that may reveal the extent of the upper atmosphere and its rate of escape. This is based on the fact that $\mathrm{CH}_{4}$ is subject to photodissociation by solar $\mathrm{H} \mathrm{Ly} \alpha$ emission, and that the lighter $\mathrm{H}$ will escape rapidly forming an extended cloud around 
Pluto. The relatively bright H Ly $\alpha$ emission (due to resonant scattering of solar emission) and the extent of the hydrogen cloud around Pluto then in principal could provide information on both the fractional abundance of atmospheric $\mathrm{CH}_{4}$ and the rate of $\mathrm{CH}_{4}$ escape (since the atomic $\mathrm{H}$ is produced in the collisional part of the atmosphere and must diffuse outward with the $\mathrm{CH}_{4}$ before escaping). However, this scenario must be modeled to determine the expected dependence of Ly $\alpha$ brightness on atmospheric parameters.

\section{MODEL CALCULATIONS}

There have been several attempts to model the structure of Pluto's extended atmosphere within various degrees of approximation based on hydrodynamic escape (Hunten and Watson 1982, Hubbard et al. 1990, McNutt 1989, Trafton et al. 1988b). The radial extent and escape rate of a hydrodynamically escaping atmosphere depend on the solar EUV flux, the composition, and the surface pressure. Regardless of how high the surface pressure is, however, the escape rate of a hydrodynamically expanding atmosphere is limited by adiabatic cooling to not exceed the solar EUV input (Hunten and Watson 1982). Hence, the exospheric structure tends to be insensitive to the details of the tropospheric structure, including low level infrared heating and any associated temperature inversion. In fact, the values derived by the different authors for a given solar EUV input fall in a fairly narrow range, so they are only mildly sensitive to the different approximations used to model the escape and our modeling of Pluto's Ly $\alpha$ emission has a low sensitivity to the choice of hydrodynamic model. On the other hand, the exospheric structure is sensitive to the value of the solar EUV input and atmospheric composition, as can be seen by comparing the analytic models of McNutt (1989). Pluto's Ly $\alpha$ distribution can therefore by expected to reflect the EUV input and atmospheric composition, providing information on each. The discovery of exobase temperatures of $700-800 \mathrm{~K}$ on Uranus and Neptune, well in excess of solar EUV heating, demonstrates that there is an additional source of energy on some planets that may strongly influence any conclusions we draw based solely on solar EUV heating. With these substantial uncertainties in mind, we will examine the implications of a class of models in which the upper atmospheric structure is determined by a predominantly $\mathrm{CH}_{4}$ composition and solar EUV heating. More sophisticated calculations can be performed when more has been learned about the composition and thermal structure of Pluto's upper atmosphere.

We use the numerical model of Trafton et al. (1988b, 1989 ) because it avoids analytical approximations in integrating the hydrodynamic equations of energy conservation and of flux, mass, and momentum continuity. Hence, it is not limited by providing a structure corresponding to an upper limit to the escape flux, as are the other models mentioned. Like the other models, this model assumes that half of the incident solar EUV flux is absorbed as heat. The thermodynamic parameters are the same as those used by Hunten and Watson (1982) but the mass and radius of Pluto have been updated to correspond to the results of the recent mutual events between Pluto and Charon, assuming these bodies have the same density. The assumed surface temperature is $50 \mathrm{~K}$, infrared heating of the atmosphere is neglected, and a pure $\mathrm{CH}_{4}$ composition is assumed. The effect of allowing another gas besides $\mathrm{CH}_{4}$, or a different value for the absorbed EUV solar flux, can be assessed by comparing McNutt's (1989) hybrid $\mathrm{CO}-\mathrm{CH}_{4}$ models with his pure $\mathrm{CH}_{4}$ case: the density above Pluto's EUV optical depth unity level is obtained from McNutt's Eqs. (12) and (14) with the parameters listed in his Tables 2 and 3. In both cases the dominant species at the altitudes discussed below is $\mathrm{CH}_{4}$ due to diffusive separation, and we therefore will examine the case of a pure $\mathrm{CH}_{4}$ upper atmosphere.

Model calculations have been performed for the case of photodissociation of $\mathrm{CH}_{4}$ by solar $\mathrm{Ly} \alpha$ emission in the hydrodynamically escaping Pluto atmosphere. We have assumed the pure $\mathrm{CH}_{4}$ hydrodynamic model of Trafton $e t$ al. (1988b) as a baseline and have superimposed the effect of photodissociation. In a hydrodynamically escaping, multispecies atmosphere, the total escape flux is driven and energetically limited by the EUV insolation absorbed and converted into heat. We consider bracketing extremes for the hydrodynamic escape of $\mathrm{H}$ : one extreme assumes that the $\mathrm{H}$ escapes in bulk with the $\mathrm{CH}_{4}$ without diffusive separation, the other case assumes that the $\mathrm{H}$ is diffusing through the hydrodynamically escaping $\mathrm{CH}_{4}$. Zahnle and Kasting (1986) have considered the supersonic (hydrodynamic) case for a light gas diffusing through a heavier, minor gas assuming a polytropic temperature distribution. They have shown that the asymptotic streaming velocities far from the planet are inversely proportional to the square root of the molecular masses. Zahnle and Kasting's result therefore suggests $\mathrm{H}$ densities, column abundances, and Ly $\alpha$ brightnesses that are $\frac{1}{4}$ of those for the bulk $\mathrm{CH}_{4}$ escape case. A hybrid variation of this case assumes that the flux of $\mathrm{H}$ diffusing through $\mathrm{CH}_{4}$ is limited by the buoyancy of $\mathrm{H}$ in $\mathrm{CH}_{4}$. This subsonic approximation (acceleration and nonlinear terms in the equations of motion are neglected) often suffices for the supersonic case as well, especially for large mass differences of the two constituents and when the total escape flux is known. In particular, this case has been found to be adequate to describe hydrodynamic mass fractionation in the atmospheres of the inner planets (Hunten et al. 1987). We also include this variation since it gives a lower limit to Pluto's $\mathrm{H}$ mixing ratio and Ly $\alpha$ intensity. 
Photodissociation of $\mathrm{CH}_{4}$ by solar $\mathrm{Ly} \alpha$ can produce atomic hydrogen by any of three paths, but $92 \%$ of the events yield two $\mathrm{H}$ atoms with a residual energy of $1.1 \mathrm{eV}$ per atom (Strobel 1985). Assigning the excess energy to kinetic energy of the $\mathrm{H}$ atoms results in a $16 \mathrm{~km}-\mathrm{sec}^{-1}$ mean speed per atom, which is highly superthermal and greater than the escape velocity at Pluto's surface $\left(v_{\mathrm{esc}}=1.2 \mathrm{~km}-\mathrm{sec}^{-1}\right)$. It is also $\sim 200$ times faster than the asymptotic streaming speed of the escaping atmosphere. The pure $\mathrm{CH}_{4}$ atmosphere becomes optically thick to incident solar Ly $\alpha$ emission at an altitude of $1.47 R_{\mathrm{P}}$, well within the collisional regime of the atmosphere, so that most of the $\mathrm{H}$ thus produced is thermalized in the neutral atmosphere. Even at the neutral temperature, $\mathrm{H}$ will escape freely from an "exobase" at $4.5 R_{\mathrm{P}}\left(v_{\mathrm{rms}}=1.4 \mathrm{~km}\right.$ $\mathrm{sec}^{-1}$ at $67 \mathrm{~K}$ and $1.6 \mathrm{~km}-\mathrm{sec}^{-1}$ at $105 \mathrm{~K}$ ), which is where the collisionless regime begins to emerge in our model. We note that the concept of the exobase loses its distinction in the hydrodynamic limit. The hydrodynamic equations can be obeyed even in the collisionless regime (e.g., the solar wind) for a suitable redefinition of the pressure, so long as some form of interaction occurs (Parker 1957). Finally, the lifetime of $\mathrm{CH}_{4}$ to photodissociation is 3-5 years at 30 $\mathrm{AU}$, so that Pluto's $\mathrm{CH}_{4}$ survives out to great distances and $\mathrm{H}$ is a minor component of the extended atmosphere.

\section{A. The H Column Abundance}

To estimate the $\mathrm{H}$ mixing ratio and column abundance in Pluto's atmosphere, we have calculated the production rate of $\mathrm{H}$ atoms in a $\mathrm{CH}_{4}$ atmosphere. The photodissociation rate of a $\mathrm{CH}_{4}$ molecule is given by

$$
J(z)=\int d \lambda \sigma_{\lambda} \Phi_{\lambda} \exp \left(-\sigma_{\lambda} N_{\mathrm{c}}\right)
$$

where $\sigma_{\lambda}$ is the cross section for photodissociation of $\mathrm{CH}_{4}$ $\left(\sigma_{\lambda}(1216 \AA)=2 \times 10^{-17} \mathrm{~cm}^{2}\right.$; Hudson 1971), $F_{\lambda}$ is the solar flux outside the atmosphere (mainly solar Ly $\alpha$ emission), and $N_{\mathrm{c}}$ is the column density of $\mathrm{CH}_{4}$ along the insolation path. We approximate the exponential factor in this integral by taking $J(z)=0$ below the height where the EUV-mean vertical optical depth $\tau=\left\langle\sigma_{\lambda}\right\rangle N^{*}$ equals unity. This height occurs where the overlying vertical column abundance of $\mathrm{CH}_{4}$ is $N^{*}=6.25 \times 10^{16}$ molecules$\mathrm{cm}^{-2}$; namely, at $1.47 R_{\mathrm{p}}$. Above this height, $J(z)=$ $6.6 \times 10^{-9}$ photodissociations per $\mathrm{CH}_{4}$ per second at Pluto. We neglect the contribution of photodissociation products to the optical depth. Since the main outcome of a photodissociation is the release of two $\mathrm{H}$ atoms, we take the production rate of $\mathrm{H}$ to be $1.3 \times 10^{-8} \mathrm{H}$ atoms per $\mathrm{CH}_{4}$ molecule per second near perihelion.

In the steady state the number of $\mathrm{H}$ atoms liberated each second below a radius $r$ in Pluto's atmosphere must equal the number passing through this radius each second.
The average flux (No. $\mathrm{H}$ atoms- $\mathrm{cm}^{-2} \mathrm{sec}^{-1}$ ) flowing outward at radius $r$ is the total number divided by the area of a shell having this radius:

$$
F_{\mathrm{H}}=\frac{2 J(r)}{4 \pi r^{2}} \int_{1.47 R_{\mathrm{P}}}^{r} d r^{\prime} 4 \pi r^{\prime 2} n\left(r^{\prime}\right)=n_{\mathrm{H}}(r) v_{\mathrm{H}}(r)
$$

where $n(r)$ is the $\mathrm{CH}_{4}$ number density, $n_{\mathrm{H}}(r)$ is the $\mathrm{H}$ number density, and $v_{\mathrm{H}}(r)$ is the $\mathrm{H}$ radial stream speed at radius $r$. The radial column abundance of $\mathrm{H}$ is thus

$$
N_{\mathrm{H}}=\int_{1.47 R_{\mathrm{P}}}^{\infty} n_{\mathrm{H}}(r) d r=\int_{1.47 R_{\mathrm{P}}}^{\infty} d r \frac{2 J}{r^{2} v_{\mathrm{H}}(r)} \int_{1.47 R_{\mathrm{P}}}^{r} d r^{\prime} r^{\prime 2} n\left(r^{\prime}\right)
$$

Since the photodissociation rate is low, the $\mathrm{CH}_{4}$ flow is nearly conserved, or

$$
4 \pi r^{2} n(r) v(r)=4 \pi R_{\mathrm{P}}^{2} F_{0}=\text { constant, }
$$

where $v(r)$ is the hydrodynamic stream speed of $\mathrm{CH}_{4}$ and $F_{0} \approx 2.1 \times 10^{10} \mathrm{~cm}^{-2}-\mathrm{sec}^{-1}$ is the flux of escaping $\mathrm{CH}_{4}$ referred to Pluto's surface. The latter is readily maintained by the sublimation of $\mathrm{CH}_{4}$ ice.

Equation (2) yields $F_{\mathrm{H}} \approx 0.10 F_{0}$ to $0.12 F_{0}$, given $n(r) \approx$ $5.1 \times 10^{10}\left(r_{0} / r\right)^{7.82} \mathrm{~cm}^{-3}$, a fit to our adopted atmosphere model valid for $r<3-4$. Hence, the escape of $\mathrm{H}$ alone will not satisfy the hydrodynamic flux required by the absorbed EUV sunlight: $\mathrm{CH}_{4}$ must also escape. This contrasts with the situation thought to have occurred in the early atmospheres of the Earth and Venus (Watson et al. 1981) where $H$ may have carried the bulk of the EUV power driving hydrodynamic escape. Therefore, we consider the bracketing extremes of bulk escape and the escape of diffusively separating species.

\section{B. Bulk Escape}

For the case where the $\mathrm{H}$ atoms escape with the same streaming speed as the $\mathrm{CH}_{4}$ molecules, $v(r)=v_{\mathrm{H}}(r)$ so the latter speed can be eliminated in Eq. (3) with the aid of Eq. (4):

$$
N_{\mathrm{H}}=\frac{2 J}{R_{\mathrm{P}}^{2} F_{0}} \int_{1.47 R_{\mathrm{P}}}^{\infty} d r n(r) \int_{1.47 R_{\mathrm{P}}}^{r} d r^{\prime} r^{\prime 2} n\left(r^{\prime}\right)
$$

With $n(r)$ obtained by power-law fits in two altitude regimes of the $\mathrm{CH}_{4}$ model, we estimate $N_{\mathrm{H}}=3.1 \times 10^{15} \mathrm{H}$ atoms-cm ${ }^{-2}$ above $1.47 R_{\mathrm{P}}$, which is $5 \%$ of $N^{*}$, the amount of $\mathrm{CH}_{4}$ in a column exposed to Ly $\alpha$. Similarly, we have integrated $n_{\mathrm{H}}(r)$ along a tangent line of sight having "impact parameter" $p$ to derive $N_{\mathrm{H}}(p)$. This result is plotted 


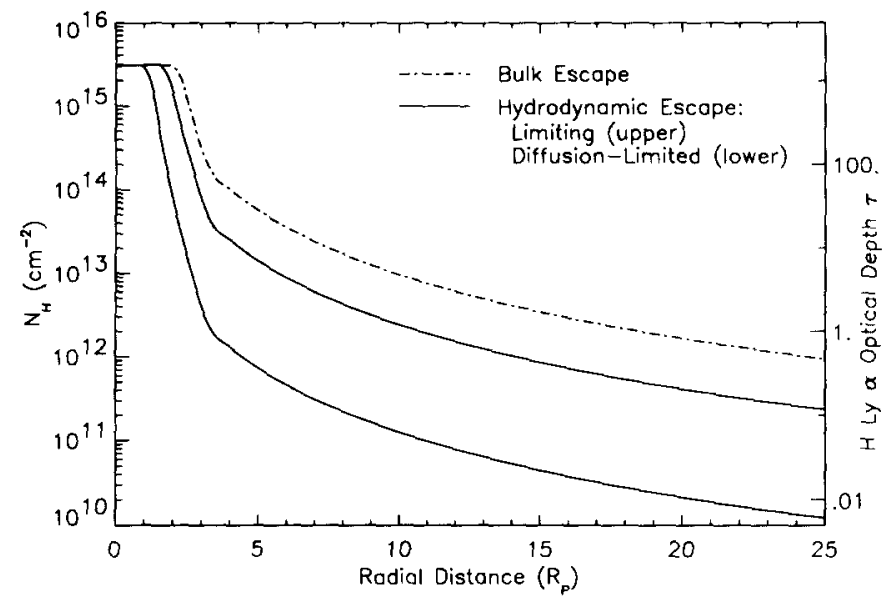

FIG. 1. Expected $\mathrm{H}$ column density as a function of tangent distance (i.e., impact parameter $p$ ) from Pluto for the conditions assumed in the text, including a pure $\mathrm{CH}_{4}$ atmosphere and thermalized diffusion of both $\mathrm{H}$ and $\mathrm{CH}_{4}$ out to $1.47 R_{\mathrm{p}}$. The effect of Charon's gravity (orbit $=16$ $R_{\mathrm{p}}$ ) is not included in this calculation.

in Fig. 1. For values of $p>1.92 R_{\mathrm{P}}$ (where the tangent atmospheric path becomes transparent to EUV light), this approximates $10 \%$ of the $\mathrm{CH}_{4}$ line-of-sight column abundance.

\section{Diffusive Separation}

Hydrogen is unlikely to escape with the same stream speed as $\mathrm{CH}_{4}$ because its mixing ratio in the bulk escape case $(\sim 10 \%)$ is large enough for buoyancy forces to cause diffusive separation (Hunten 1973) above $1.5 R_{\mathrm{P}}$. Above $1.5 R_{\mathrm{P}}$, which is very close to the base of the photodissociation zone, the mixing ratio of $\mathrm{H}$ increases to a value greater than Pluto's critical value $\sim 0.0018$ : at these altitudes the diffusion rate is no longer source-limited but limited by the rate of diffusion processes. An equivalent statement is that the crossover mass (Hunten et al. 1987) tends to fall below the molecular weight of $\mathrm{CH}_{4}$ above this radial distance, causing $\mathrm{H}$ to diffuse outward through the $\mathrm{CH}_{4}$. Therefore, we adopt the scenario where both $\mathrm{H}$ and $\mathrm{CH}_{4}$ are escaping hydrodynamically, but the $\mathrm{H}$ is escaping faster because it is diffusing through the $\mathrm{CH}_{4}$. Since the total escape flux is constrained, the higher $\mathrm{H}$ stream speed will reduce the $\mathrm{H}$ density, the $\mathrm{H}$ column abundance, and the predicted Ly $\alpha$ brightness relative to the bulk escape case, where both species have the same stream velocity.

We therefore set the crossover mass (e.g., Eq. (16) of Hunten et al. 1987) to be equal to the mass of a $\mathrm{CH}_{4}$ molecule to improve our estimate of the $\mathrm{H}$ mixing ratio. This hybrid approach, which utilizes the subsonic approximation and neglects accelerations, gives a revised asymptotic mixing ratio (independent of height above $-1.9 R_{\mathrm{p}}$ ):

$$
f_{1}=\left[\frac{\left(\mu_{\mathrm{C}}-\mu_{\mathrm{H}}\right) m_{\mathrm{P}} b g_{0}}{k T F_{\mathrm{Ho}}}-1\right]^{-1},
$$

where $\mu_{i}$ is the molecular weight of species $i\left(m_{\mathrm{P}}=\right.$ proton mass), $b$ is the diffusion parameter, $g_{0}=66 \mathrm{~cm}^{-\mathrm{sec}^{-2}}$ is Pluto's surface gravity, and $F_{\mathrm{Ho}} \approx 0.12 F_{0}$ is the flux of escaping $\mathrm{H}$ above $\sim 2 R_{\mathrm{P}}$ referred to Pluto's surface, estimated from Eq. (2). For a temperature range of 50 to $100 \mathrm{~K}$, we estimate $b \approx(0.74$ to 1.2$) \times 10^{19} \mathrm{~cm}^{-1}-\mathrm{sec}^{-1}$ on the basis of $\mathrm{H}-\mathrm{N}_{2}$ and $\mathrm{H}-\mathrm{O}_{2}$ data (Banks and Kockarts 1973) so that a crossover mass of $16 m_{\mathrm{P}}$ requires a mixing ratio for $\mathrm{H}$ of 0.0014 to 0.0018 . This permits the $\mathrm{H}$ flux to be diffusion-limited yet allows the $\mathrm{CH}_{4}$ to be dragged along and also escape. The $\mathrm{H}$ mole fraction then corresponds to about $1.3 \%$ of the bulk escape value, so that the predicted intensities are $1.3 \%$ of those predicted by the bulk escape case. This more realistic, hybrid case is illustrated by the lower solid line in Fig. 1. Also plotted in Fig. 1 is the limiting hydrodynamic case, for which the $\mathrm{H}$ density, column abundance, and Ly $\alpha$ brightness are ${ }_{4}^{1}$ of those for the bulk escape case. In conclusion, we favor the regime bracketed by the models predicting $1.3 \%$ and $25 \%$ of the bulk escape brightnesses (solid lines in Fig. 1).

\section{Modeling Results}

The results of modeling the $\mathrm{CH}_{4}$ and $\mathrm{H}$ diffusion and escape are presented in Fig. 1 and Table I. Figure 1 shows the integrated column of $\mathrm{H}$ atoms as a function of impact parameter from Pluto above the $\tau=1$ level for $\mathrm{CH}_{4}$ photoabsorption for the bracketing cases. The column $N_{\mathrm{H}}$ has an upper limit of $3.1 \times 10^{15} \mathrm{~cm}^{-2}$ corresponding to $\tau=1$ in $\mathrm{CH}_{4}$, since the bulk escape case (but not the hydrodynamic limit) has the $\mathrm{H}$ atoms thermalized with the $\mathrm{CH}_{4}$ (i.e., a nearly constant $\mathrm{H} / \mathrm{CH}_{4}$ ratio above $1 R_{\mathrm{P}}$ ). The column then falls off rapidly with increasing altitude out to $4.5 R_{\mathrm{P}}$, where the atmosphere becomes collisionally thin. Above this altitude $N_{\mathrm{H}}$ is further reduced by the ratio of the (thermal velocity/streaming velocity) of the $\mathrm{H}$ atoms. The $\mathrm{H}$ atmosphere above $4.5 R_{\mathrm{P}}$ is dominated by the slower streaming $\mathrm{H}$ atoms from lower in the atmosphere rather than by faster atoms created in situ. The $\mathrm{H}$

TABLE I

Adopted $\mathrm{CH}_{4}$ Densities and Streaming Velocities

\begin{tabular}{ccc}
$\begin{array}{c}\text { Plutocentric } \\
\text { distance }\left(R_{\mathrm{p}}\right)\end{array}$ & $\begin{array}{c}\text { Streaming velocity } \\
(\mathrm{km} / \mathrm{sec})\end{array}$ & $\begin{array}{c}\mathrm{CH}_{4} \text { atmospheric } \\
\text { density }\left(\mathrm{cm}^{-3}\right)\end{array}$ \\
\hline 1.5 & 0.000035 & $2.8 \times 10^{9}$ \\
1.86 & 0.0002 & $3.0 \times 10^{8}$ \\
4.0 & 0.007 & $1.9 \times 10^{6}$ \\
5.0 & 0.01 & $7.5 \times 10^{5}$ \\
6.0 & 0.02 & $3.6 \times 10^{5}$ \\
\hline
\end{tabular}


Ly $\alpha$ optical depth for line center resonant scattering of solar Ly $\alpha$ emission corresponding to $N_{\mathrm{H}}$ is plotted on the right axis in Fig. 1.

One interesting result is that the $\mathrm{H}$ streaming flux is relatively independent of the fraction of $\mathrm{CH}_{4}$ in the atmosphere, as long as the $\mathrm{CH}_{4}$ is optically thick to photolysis by solar $\mathrm{Ly} \alpha . \mathrm{A} \mathrm{CH}_{4}$ mixing ratio as low as $10^{-6}$ would satisfy this criterion, as long as the dominant constituent is optically thin at Ly $\alpha$. Besides $\mathrm{H}$, molecular hydrogen will also be released in the photodissociation of $\mathrm{CH}_{4}$. However, since its flux cannot exceed that of H, Eq. (6) implies that it will also be a minor constituent and that the $\mathrm{CH}_{4}$ structure is not materially affected. As for $\mathrm{H}$, the mixing ratio of $\mathrm{H}_{2}$ should be essentially inversely proportional to the flux of escaping $\mathrm{H}_{2}$ for the range of conditions in Pluto's atmosphere. We have not considered the effect of another bulk gas, such as $\mathrm{CO}$ or $\mathrm{N}_{2}$, on the atmospheric structure because this would require a more involved hydrodynamic model which is beyond the scope of this paper. A greater uncertainty is the total energy input for upper atmospheric heating: until the source of thermospheric heating on the giant planets is understood, we can only assume that the solar EUV flux is a lower limit to the upper atmosphere heating on Pluto.

\section{IUE OBSERVATIONS}

Due mainly to the small angular size of Pluto (roughly 0.1 arcsec diameter) compared with the point spread function of IUE $(5 \mathrm{arcsec})$ the sensitivity of IUE to detect $\mathrm{H}$ Ly $\alpha$ emission was expected to be very low, and no such observations were performed in the first 12 years of IUE operations. However, the emerging view of Pluto as having an extended atmosphere containing a large amount of $\mathrm{CH}_{4}$ suggests the possibility of an $\mathrm{H}$ cloud closer to the size of the IUE angular resolution, in which case the IUE might be able to detect a signal on the order of $100 R$. Although the IUE sensitivity is not optimal, we undertook observations to test the theory and to detect or constrain the possible extent of Pluto's atmosphere.

IUE short wavelength prime (SWP) spectrograph observations of Pluto were performed over 24-25 July 1989 (see Table II), with Pluto observed as a point source in the large aperture of the SWP. The observing technique was the same as for Uranus and Neptune, previously described by Clarke et al. (1986), in which Pluto is observed in the antisolar direction and spatially resolved above the geocoronal (GEO) and interplanetary medium (IPM) emissions. Although the GEO and the great majority of the IPM emissions are in the foreground of Pluto, there is also a background component of the IPM on the order of 100 rayleighs that may be produced beyond the line of sight to Pluto. This emission will be occulted by the physical disk of Pluto, but with a line of sight Doppler
TABLE II

IUE Observations of Pluto

\begin{tabular}{clcc}
\hline $\begin{array}{c}\text { SWP } \\
\text { image no. }\end{array}$ & Object & $\begin{array}{c}\text { Length } \\
\text { (min) }\end{array}$ & $\begin{array}{c}\text { Background 4 } I \\
\text { (krayleigh) }\end{array}$ \\
\hline 36727 & Sky & 30 & 5.65 \\
36728 & Pluto & 30 & 2.26 \\
36729 & Sky & 60 & 1.77 \\
36730 & Pluto & 90 & 1.46 \\
36731 & Pluto & 120 & 1.37 \\
36732 & Sky & 90 & 1.35 \\
36734 & Sky & 90 & 1.63 \\
\hline
\end{tabular}

Upper Limits to Pluto H Ly a Emission

\begin{tabular}{ccc}
$\tau=1$ tangent & $\begin{array}{c}\text { Emitting region } \\
\text { angular diameter } \\
\text { height }\left(R_{\mathrm{P}}\right)\end{array}$ & $\begin{array}{c}1 \sigma 4 \pi I \text { upper } \\
\text { limit (rayleigh) }\end{array}$ \\
\hline 52 & 5.5 & 70 \\
38 & 4 & 130 \\
19 & 2 & 520 \\
9.4 & 1 & 2100 \\
4.7 & 0.5 & 8300 \\
\hline
\end{tabular}

shift of nearly $20 \mathrm{~km} / \mathrm{sec}$ at Pluto's present position this emission will not be strongly absorbed above the $\tau=1$ level for $\mathrm{CH}_{4}$ absorption in Pluto's atmosphere at $1.5 R_{\mathrm{P}}$. This implies a region roughly 0.15 arcsec in diameter that is opaque to the background IPM emission, compared with an $\mathrm{H}$ emitting region that is $6-10 R_{\mathrm{P}}$ or $0.3-0.5$ arcsec in diameter (FWHM) and transparent to the IPM background. For observations in which Pluto's emission cloud is not resolved, we would expect the cloud to appear superimposed on an IPM emission reduced by 9 to $25 \%$ due to the central obscuration of the background IPM. Depending on the relative brightnesses of Pluto and the background IPM, Pluto might appear as either an emission or an absorption in a spatial image: for the predicted 60 rayleigh Pluto central brightness and an IPM background of 200-300 rayleighs, we would expect Pluto to appear either nearly equal to or greater than the surrounding sky emission. If Pluto's emitting region exceeds a radius of 4 $R_{\mathrm{P}}$, Pluto would definitely appear as an emission feature assuming these brightnesses.

No H Ly $\alpha$ emission (in either emission or absorption) has been detected from Pluto within the observational uncertainties. The IUE sensitivity to Pluto's emission is determined mainly by the background brightness and the angular size of the emitting region. The measured $1 \sigma$ uncertainty for an emitting region the size of the IUE point spread function (PSF FWHM $=5.5 \mathrm{arcsec}$ ) is $5.3 \%$ of the background brightness when the background and planetary measurements are obtained during the same shift (determined empirically by Clarke et al. 1986). The back- 


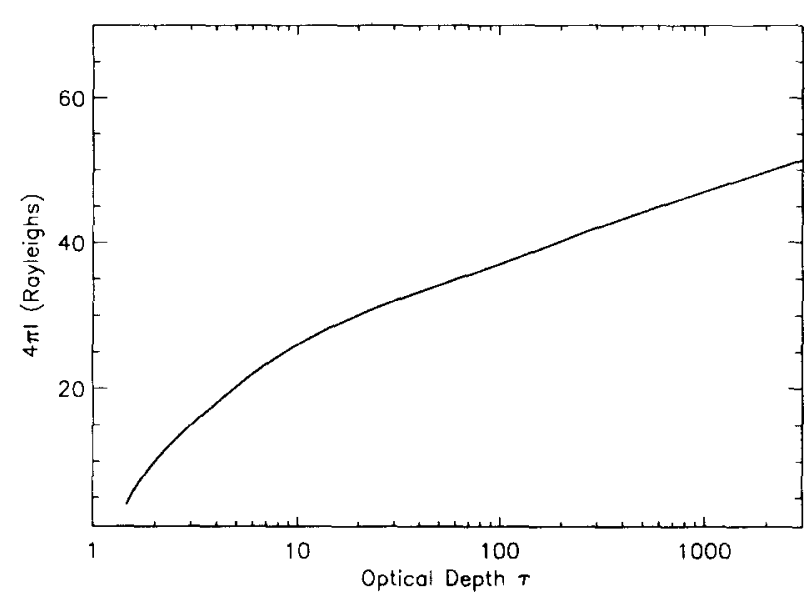

FIG. 2. Calculated H Ly $\alpha$ emission curve of growth for resonant scattering of solar emission, corresponding to the $\mathrm{H}$ column values in Fig. 1.

ground brightness, due mainly to the interplanetary Ly $\alpha$ emission (Pluto was roughly $20^{\circ}$ from the inflow direction), reached a minimum of 1300 rayleighs during these observations. The solar Ly $\alpha$ flux at $1 \mathrm{AU}$ is estimated to have been $4 \pm 1 \times 10^{11} \mathrm{ph}-\mathrm{cm}^{-2}-\mathrm{sec}^{-1}$ based on the observed solar $\mathrm{Ca}(\mathrm{II})$ flux at the time of these observations (G. Rottman, personal communication). Scattering of the interplanetary emission from Pluto's atmosphere is unlikely to contribute substantially to the subsolar brightness, because the $21 \mathrm{~km}-\mathrm{sec}^{-1}$ velocity of the interplanetary medium Doppler shifts the interplanetary line well off the Pluto line at the present orbital position of Pluto.

\section{BRIGHTNESS CALCULATIONS}

Resonant scattering of solar H Ly $\alpha$ emission has been modeled by the application of Chandrasekhar $X$ and $Y$ functions to a plane parallel scattering layer, assuming a measured solar line profile and approximating the absorption by $\mathrm{CH}_{4}$ with a completely absorbing lower boundary (Clarke et al. 1986). Based on our experience with this simple model and other models employing realistic model atmospheres on the other outer planets, we expect that our results are accurate to within a factor of 2 . The temperature of Pluto's upper atmosphere (in the range 1.4-5 $R_{\mathrm{p}}$ ) is not known, but is likely to be somewhat higher than the 67-105 K of the middle atmosphere due to a combination of solar EUV and plasma heating. We assume an isothermal upper atmosphere at $150 \mathrm{~K}$ for these calculations: an improved temperature value will improve the accuracy of these estimates. Results are plotted in Fig. 2, with the $\mathrm{H}$ optical depths corresponding to the tangential distances from Pluto plotted in Fig. 1. The central optically thick "cloud" about Pluto is 60 rayleighs in brightness, and an extended emission region of 20-60 rayleighs extends out to roughly 3-8 $R_{\mathrm{P}}$. The best discriminant between the different escape models is seen to be the brightness of the extended emission, and not the brightness of the central cloud.

Since an unresolved emission is blurred to the IUE PSF by the instrument, the sensitivity of IUE to a smaller emission region is decreased by the ratio of areas $\left(R_{\mathrm{e}} / R_{\mathrm{i}}\right)^{2}$, where $R_{\mathrm{e}}=$ FWHM of the emitting region and $R_{\mathrm{i}}=$ FWHM of the PSF ( $5.5 \operatorname{arcsec}$ ). From Table II we derive a limiting $1 \sigma$ sensitivity of 70 rayleighs from a 5.5 -arcsec cloud, and this limit is scaled by the size of the emitting region in Table II. The sensitivity in spatial maps can be improved upon chiefly by applying higher angular resolution and a detector with a larger dynamic range (IUE exposures were limited to less than $2 \mathrm{hr}$ due to saturation on the background Ly $\alpha$ emission). Although the interplanetary background should decrease by a factor of 2-3 near solar minimum in 1996, Pluto's brightness should decrease by a similar factor, so that the expected contrast between Pluto and the background emission will not improve substantially. A limiting sensitivity of 10 rayleighs (i.e., a signal to noise ratio of 150 ) with an angular resolution of $2-4 R_{\mathrm{P}}$ would tightly constrain the Ly $\alpha$ brightness and extent of Pluto's atmosphere. Although this would be a difficult measurement, observations of the extended $\mathrm{CH}_{4}$ (or $\mathrm{N}_{2}$ or $\mathrm{CO}$ ) atmosphere are likely to be more difficult due to the much weaker emissions from these species. Finally, it is important to note that Pluto's Ly $\alpha$ emission is not coincident in wavelength with the GEO and IPM emissions. The IPM emission is Doppler shifted by $0.08 \AA$ blueward of Pluto's line center, and the GEO emission may be shifted either red or blue by $0.12 \AA$ from the Earth orbital velocity at the appropriate times of year. Observations with $0.04-\AA$ spectral resolution and an angular resolution of 5-10 $R_{\mathrm{P}}$ could detect Pluto's Ly $\alpha$ emission spectrally resolved from the sky emissions without the high sensitivity requirements of spatial maps.

\section{COMPARISON WITH TRITON}

H Ly $\alpha$ emission from Triton was measured by the Voyager 2 UVS in 1989 (Broadfoot et al. 1989), with a subsolar point brightness of $\sim 120$ rayleighs. Although Triton and Pluto are believed to have qualitatively similar atmospheres, the $\mathrm{CH}_{4}$ in Triton's atmosphere $\left(N \approx 2 \times 10^{16} \mathrm{~cm}^{-2}\right)$ has an optical depth for photolysis by solar Ly $\alpha$ of only $\tau \approx 0.1$. The effect of rayleigh scattering by $\mathrm{N}_{2}$ is small, so the surface albedo on Triton may contribute significantly to the observed emission. Triton's brightness at $\mathrm{H}$ Ly $\alpha$ relative to Pluto may also be due in part to bombardment of Triton's atmosphere by trapped charged particles in Neptune's magnetosphere. Evidence for this is the $\sim 60$ - to 100-rayleigh 
emission seen on Triton's night side, compared with the 70- to 120-rayleigh daytime brightness (Ben Jaffel and Yelle 1990). The plasma heating also dominates the effects of solar heating in Triton's atmosphere. We therefore conclude that our predicted 60-rayleigh brightness for the subsolar point on Pluto is not inconsistent with the Voyager observations of Triton.

\section{CONCLUSIONS}

We have calculated the production and both bulk and separated hydrodynamic escape rates of hydrogen from Pluto's upper atmosphere, and compared the calculated distributions of Pluto's hydrogen atmosphere with observational upper limits to $\mathrm{H}$ Ly $\alpha$ emission obtained with the IUE Observatory. The validity of these calculations depends on the assumptions that $\mathrm{CH}_{4}$ is the dominant constituent above 1.4 $R_{\mathrm{P}}$, and that solar EUV is the main source of thermospheric heating. We find that Pluto is likely to have a very substantial extended hydrogen atmosphere, and that the extent and density of this atmosphere should depend much more on the nature of the escape process than on the fraction of $\mathrm{CH}_{4}$ in the atmosphere. $\mathrm{H}$ Ly $\alpha$ emission from resonant scattering should provide the most easily observable signature of this extended atmosphere until in situ data can be obtained.

\section{ACKNOWLEDGMENTS}

We acknowledge support from the IUE Observatory staffs at VILSPA and GSFC, assistance from the RDAF staff at the University of Colorado in the acquisition and reduction of the IUE data, and support from NASA Grants NAGW-1766 to the University of Michigan, NAG5-1151 to the University of Colorado, and NAGW-1477 to the University of Texas.

\section{REFERENCES}

Banks, P. M., and G. Kockarts 1973. Aeronomy, Part B. Academic Press, New York.

Ben JAfFel, L., AND R. V. Yelle 1990. Analysis of Triton's Lyman alpha emissions. Bull. Am. Astron. Soc. 22, 1127.
BROADFOOT, A. L. et al. 1989. Ultraviolet spectrometer observations of Neptune and Triton. Science 246, 1459.

Clarke, J. T. et al. 1986. Continued observations of the H Ly $\alpha$ emission from Uranus. J. Geophys. Res. 91, 8771.

Elliot, J. L. et al. 1989. Pluto's atmosphere. Icarus 77, 148.

Hubbard, W. B., D. M. Hunten, S. W. Dieters, K. M. Hill, and R. D. WATSON 1988. Occultation evidence for an atmosphere on Pluto. Nature 336, 452.

Hubbard, W. B., R. V. Yelle, and J. I. Lunine 1990. Nonthermal Pluto atmosphere models. Icarus 84, 1.

Hudson, R. D. 1971. Critical review of UV photoabsorption cross sections for molecules of astrophysical and aeronomic interest. Rev. Geophys. 9, 305.

HUNTEN, D. M. 1973. The escape of light gases from planetary atmospheres. J. Atmos. Sci. 30, 1481.

Hunten, D. M., and A. J. Watson 1982. Stability of Pluto's atmosphere. Icarus 51, 665.

Hunten, D. M., R. O. Pepin, and J. C. G. Walker 1987. Mass fractionation in hydrogenic escape. Ic arus $69,532$.

PARKer, E. 1957. Newtonian development of the dynamical properties of ionized gases of low density. Phys. Rev. 107, 924.

MCNutT, R. L. 1989. Models of Pluto's upper atmosphere. Geophys. Res. Lett. 16, 1225.

Stern, S. A., And L. M. Trafton 1984. Constraints on bulk composition, seasonal variation, and global dynamics of Pluto's atmosphere. Icarus 57, 231.

Strobel, D. F. 1985. Photochemistry of the atmospheres of the outer planets and their satellites. In The Photochemistry of Atmospheres (J. S. Levine, Ed.), p. 394. Academic Press.

Trafton, L. M. 1989. Pluto's atmosphere near perihelion. Geophys. Res. Lett. 16, 1213.

Trafton, L. M., S. A. Stern, and G. R. Gladstone 1988a. The Pluto-Charon system: The escape of Charon's primordial atmosphere. Icarus 74, 108.

Trafton, L., A. L. Whipple, AND S. A. STERN 1988b. The extended atmosphere of the Pluto-Charon System. Bull. Amer. Astron. Soc. 19, 1071.

Trafton, L., A. L. Whipple, AND S. A. SteRn 1989. Hydrodynamic calculations of Pluto's atmosphere. Ninth Planet New's 7.

Watson, A. J., T. M. Donahue, and J. C. G. Walker 1981. The dynamics of a rapidly escaping atmosphere. Applications to the evolution of Earth and Venus. Icarus 48, 150.

ZahNlE, K. J., AND J. F. Kasting 1986. Mass fractionation during transonic hydrodynamic escape and implications for loss of water from Venus and Mars. Icarus 68, 462. 